\title{
Moringa oleifera Lam prevents acetaminophen induced liver injury through restoration of glutathione level
}

\begin{abstract}
Initiation of acetaminophen (APAP) toxicities is believed to be promoted by oxidative stress during the event of overdosage. The aim of the present study was to evaluate the hepatoprotective action of Moringa oleifera Lam (MO), an Asian plant of high medicinal value, against a single high dose of APAP. Groups of five male Sprague-Dawley rats were pre-administered with MO (200 and $800 \mathrm{mg} / \mathrm{kg}$ ) prior to a single dose of APAP (3 g/ $/ \mathrm{kg}$ body weight; p.o). Silymarin was used as an established hepatoprotective drug against APAP induced liver injury. The hepatoprotective activity of MO extract was observed following significant histopathological analysis and reduction of the level of alanine aminotransferase (ALT), aspartate aminotransferase (AST) and alkaline phosphatase (ALP) in groups pretreated with MO compared to those treated with APAP alone. Meanwhile, the level of glutathione (GSH) was found to be restored in MO-treated animals compared to the groups treated with APAP alone. These observations were comparable to the group pretreated with silymarin prior to APAP administration. Group that was treated with APAP alone exhibited high level of transaminases and ALP activities besides reduction in the GSH level. The histological hepatocellular deterioration was also evidenced. The results from the present study suggested that the leaves of MO can prevent hepatic injuries from APAP induced through preventing the decline of glutathione level.
\end{abstract}

Keyword: Acetaminophen; Paracetamol; Glutathione; Hepatoprotective; Hepatotoxicity 